\title{
Percutaneous versus surgical drainage of malignant pericardial effusion: Still no tiebreaker
}

\author{
Robbin G. Cohen, MD, MMM
}

\section{See related article on pages 2288-93.}

The optimal treatment strategy for patients with malignant pericardial effusion poses a multidimensional surgical challenge because of the advanced nature of the neoplastic disease and its associated poor prognosis (median life expectancy of approximately 15 weeks). ${ }^{1}$ Important variables include the type of malignancy and its potential for treatment with systemic or radiation therapy, as well as the general condition and hemodynamic stability of the patient. Successful therapeutic modalities have included pericardiocentesis with short- and long-term catheter drainage, percutaneous drainage with intrapericardial sclerosis, percutaneous balloon pericardiotomy, and surgical drainage with pericardial window. ${ }^{2}$ Most patients referred for surgery seem to be those with ongoing or precardiac tamponade, necessitating urgent or emergency drainage. There are no prospective, randomized trials comparing these therapeutic modalities with respect to success rate or long-term outcome. What is clear regarding the evaluation and treatment of patients with malignant pleural effusions is that their care should take place in a multidisciplinary setting, with input from medical and radiation oncologists, radiologists, cardiologists, oncologic support staff, and surgeons.

In this issue of the Journal, Çelik and colleagues ${ }^{3}$ from Italy present their retrospective review of data from the International Neoplastic Pericarditis Treatment Study that compared the results of chemotherapy alone, chemotherapy plus percutaneous drainage, and chemotherapy plus surgical drainage with pericardial window (through a left anterior thoracotomy) in patients with proven malignant pericardial effusions. Their data set included 175 patients treated in 8 centers throughout 3 countries. The study is unique in that it claims to be the first of its kind to compare the results of percutaneous drainage with surgical drainage

From the Division of Cardiothoracic Surgery, USC/Keck School of Medicine, Los Angeles, Calif.

Disclosures: Author has nothing to disclose with regard to commercial support.

Received for publication Sept 14, 2014; accepted for publication Sept 15, 2014

Address for reprints: Robbin G. Cohen, MD, MMM, Division of Cardiothoracic Surgery, Keck/USC School of Medicine, 1520 San Pablo St \#4300, Los Angeles, CA 90033 (E-mail: rcohen@usc.edu).

J Thorac Cardiovasc Surg 2014;148:2294-5

0022-5223/\$36.00

Copyright (c) 2014 by The American Association for Thoracic Surgery

http://dx.doi.org/10.1016/j.jtcvs.2014.09.033 and pericardial window in the setting of chemotherapy and to suggest both a response and survival advantage with the latter. The message is weakened by the study's retrospective nature, as well as the absence of an enrollment protocol in that patients were treated according to "institutional preference."

When determining response rate and survival advantage after drainage for malignant pericardial effusion, it is important to place the indications for intervention into perspective. If we assume that drainage of a malignant pericardial effusion has virtually no oncologic impact, then the most effective therapy is the one that gives the best physiologic response (complete drainage), yields the lowest recurrence rate, and is best tolerated by the patient. Çelik and colleagues $^{3}$ have shown that both pericardiocentesis with catheter drainage and surgical drainage with pericardial window are well tolerated by a group of patients who tend to be debilitated by virtue of their advanced malignancies. Although response rate and survival were better with the surgical approach in this study, Çelik and colleagues ${ }^{3}$ did not really attempt an explanation, nor have they made a strong recommendation for the surgical approach. Although somewhat disappointing, this is probably appropriate.

One would expect that the surgical approach would yield more complete pericardial drainage; however, that did not turn out to be true in this study. Although total response (complete response group plus partial response group) was superior in the surgical group, complete response was almost identical between the 2 groups $(29.3 \%$ for chemotherapy with percutaneous drainage and $31.1 \%$ for chemotherapy with a pericardial window). The survival advantage associated with the surgical approach also goes unexplained. Was it due to a lower recurrence rate related to the surgical approach and pericardial window, or could it be that the surgical group had a higher number of patients with malignancies other than lung cancer who went on to have a response to systemic therapy? The unfortunate reality is that the future of successful treatment of malignant pericardial effusions lies not in the hands of cardiologists or thoracic surgeons, but in those of the medical and radiation oncologists who have yet to successfully treat these advanced malignancies.

Are the results of Çelik and colleagues ${ }^{3}$ compelling enough to make a case for surgical drainage plus pericardial window instead of percutaneous drainage, all in the setting of chemotherapy? Probably not. Only a prospective, randomized trial with clear-cut advantages would do that. Furthermore, percutaneous therapy can be effective and 
more practical, especially in the presence of cardiac tamponade. That said, there are some situations in which the surgical approach is clearly preferable. If the diagnosis is in doubt (as many as $30 \%$ of patients with pericardial effusion and a known malignancy actually have benign pericardial effusions as a result of chemotherapy, radiation therapy, autoimmune disease, or infection), or percutaneous drainage is technically difficult, then the surgical approach has clear advantages. Beyond this, it will be the oncologic advances in the treatment of these advanced malignancies that will offer the real hope for improved results in the future.

\section{References}

1. Gornik H, Gerhard-Herman M, Beckman J. Abnormal cytology predicts poor prognosis in cancer patients with pericardial effusion. J Clin Oncol. 2005;23:5211-6.

2. Burazor I, Imazio M, Markel G, Adler Y. Malignant pericardial effusion. Cardiol ogy. 2013;124:224-32.

3. Çelik S, Lestuzzi C, Cervesato E, Dequanter D, Piotti P, De Biasio M, et al Systemic chemotherapy in combination with pericardial window has better outcomes in malignant pericardial effusions. J Thorac Cardiovasc Surg. 2014 148:2288-93. 